\title{
Are Lower Extremity Injuries Related to Spinal form Abnormalities in Professional Football Players? A Prospective Cohort Study
}

\author{
Sara Lotfian, ${ }^{1}$ Navid Moghadam, ${ }^{1,2,}$ Bahar Hassnamirzaie, ${ }^{2,3,4}$ and Salman Khalifeh Soltani ${ }^{1,2,3}$ \\ ${ }^{1}$ Sports and Exercise Medicine Department, Rasool Akram Hospital, Iran University of Medical Sciences, Tehran, Iran \\ ${ }^{2}$ Sports Medicine Research Center, Neuroscience Institute, Tehran University of Medical Sciences, Tehran, Iran \\ ${ }^{3}$ Iran Football Medical Assessment and Rehabilitation Center (IFMARC), Tehran, Iran \\ ${ }^{4}$ Sports and Exercise Medicine Department, Shahid Beheshti University of Medical Sciences, Tehran, Iran \\ "Corresponding author: Navid Moghadam, Rasool Akram Hospital, Niyayesh St, Sattarkhan Ave, Tehran, Iran. Tel: +98-935 254 2280, E-mail: navid.mgd@gmail.com
}

Received 2017 June 18; Revised 2017 July 11; Accepted 2017 August 26.

\begin{abstract}
Background: A professional football player sustains 2 injuries per season on average. Lower extremity injuries are the most common injuries among football players. To develop a prevention programs it is essential to recognize risk factors associated with the incidence of injuries. Previous studies focused on intrinsic risk factors of lower extremity. The association between spine abnormities and lower extremity injuries in professional athletes have only been scarcely studied.

Objectives: The purpose of this study was to evaluate the effects of various spinal form abnormalities on the rate of lower extremity injuries in male professional football players.

Methods: This cohort study among Iranian Football players was implemented during the 2015 - 2016 season. All players were assessed for spinal alignment during Pre Participation Examinations. In total, club doctors documented information of 244 players out of 420 players.

Results: There were 155 injuries recorded in total with at least one day absence from training or match, of which the most common injuries were hamstring muscle injuries $(n=41,26 \%)$, ankle ligament injuries $(n=34,22 \%)$ and adductor injuries $(n=25,16 \%)$ respectively. Increasing thoracic kyphosis Cobb's angle was associated with a reduction in the likelihood of exhibiting quadriceps injury, odds ratio (CI 95\%): 0.806 (0.671 - 0.968).

Conclusions: The reason why players with lower thoracic kyphosis Cobb's angle may be at risk for quadriceps injury is not clear. But biomechanical changes such as extensibility of hamstring and the balance between hamstring and quadriceps may partially explain the increased risk.
\end{abstract}

Keywords: Soccer, Athletic Injuries, Spinal Curvatures

\section{Background}

Federation internationale de football association (FIFA) estimated the number of football players to be 265 million worldwide, which makes it the most common played sport in the world (1). Among all football players, it is expected that 200 thousand persons play professional and semi-professional football (2). A professional player sustains 2 injuries per season on average (3). Lower extremity injuries are the most common injuries among football players and FIFA estimates that 30 billion dollars a year is spent for management of injured football players (4).

To develop prevention programs it is essential to recognize risk factors associated with the incidence of injuries. Previous studies focused on intrinsic risk factors of lower extremity including: previous injury, (5-7) poor flexibility, $(5,8,9)$ and declined muscle strength or imbalance in strength of antagonist muscle groups $(10,11)$ but there is a clear kinematic relationship between spine and lower extremity movement (12). The association between spine abnormities and lower extremity injuries in professional athletes have only been scarcely studied (13). It has been shown that various abnormalities in the spine such as lumbar hyper lordosis may influence general injury rates of lower extremity parts like knee, ankle and muscles. But sub analyses of lower extremity injuries have not been reported in professional football players.

The purpose of this study was to evaluate the effects of various spinal form abnormalities on the rate of lower extremity injuries in male professional football players.

\section{Methods}

\subsection{Study Sample and Study Period}

This prospective cohort study is a part of a larger group of risk factor-injury surveillance studies carried out at Iran's Football Medical assessment and rehabilitation center (IFMARC). This cohort study among Iranian Football players was implemented during the 2015 - 2016 season (September to May). All 16 clubs of premier football league were asked to participate in the study during Pre Participation Examinations and they all agreed. Signed informed consents were obtained from all included players individually. The examinations and assessments of all teams were done in IFMARC, Tehran, Iran. All 16 club doctors were trained to document injuries and return-to-play of football players in an online platform. All matches of premier league were held on natural grass fields (14).

All players were assessed for spinal alignment during Pre Participation Examinations. In total, club doctors documented information of 244 players out of 420 players. 
During the season, club doctors failed to report information of 176 players because of transfer or difficulties in dealing with online reporting platform.

The study proposal underwent an ethical review and was approved by the ethics committee of Iran University of medical Sciences and the Iran's football federation Medical committee.

\subsection{Baseline Examinations and Assessment}

\subsubsection{Examinations}

The study protocol included an examination of each player, in accordance to Part 6.1: Spinal column and pelvic level of pre-competition Medical assessment (PCMA). The Federation internationale de football association (FIFA) has developed and standardized PCMA and applied it at FIFA World Cups (15). All the examinations were done by Board certified Sports and exercise medicine specialists.

The examination comprised of evaluation of spine form, pelvic level, sacroiliac joint, cervical spine range of motion and spinal flexion (stand and reach test) (Table 1). The examinations were carried out according to the instruction of F-MARC football medicine manual, 2nd edition (16).

\subsubsection{Assessment}

Sagittal curvature of thoracic and lumbar parts of spine were measured in the relaxed standing position using a Spinal Mouse system (Idiag, Fehraltdorf, Switzerland) and stated as Cobb's angle (17). Spinal Mouse is a computer-based measuring device, which assesses sagittal spinal inter-segmental angles with a non-invasive surfacebased technique. Validity and reliability of Spinal Mouse to measure the spinal curvatures were reported before (18).

\subsection{Injuries}

The Club doctors were responsible for recording all injuries resulting in a player being unable to fully join training or match play. The record provided data of date, site and type of injury. Each injury was tracked until the final day of return to play (training or match). The injuries were classified into two categories of severity based on the duration of absence from training and match sessions: minor ( $<28$ days) and major ( $\geq 28$ days). The definition of injury and severity were based on the consensus statement for football injury surveillance (19).

In the present cohort study, only lower extremity injuries were included (Box 1). The registration of an injury was based on a clinical examination by the club doctor. Premier league club doctors were previously trained based on F-MARC football medicine manual, 2nd edition (16).

\subsection{Statistical Analyses}

Relative risks (RRs) with 95\% CIs are presented for association of each independent categorical variable with occurrence of each injury (total and major injuries). Continuous independent variables were analyzed for association with each injury (total and major injuries) using binary logistic regressions (Enter method).

Categorical Independent variables were spine form (thoracic, lumbar, scoliosis) and sacroiliac joint statues. Continuous independent variable included thoracic kyphosis Cobb's angle, lumbar lordosis Cobb's angles, cervical spine range of motion and spinal flexion test results. Occurrence of injuries were considered as significance level was set at P value $<0.05$.

\section{Results}

\subsection{Players and Baseline Examinations and Assessments}

There were 244 players included. Baseline examination results of these 244 players are presented in Table 2. In total, 54 (22.1\%) players showed abnormal thoracic sagittal curve (hyper kyphosis or straight back), 63 (25.8\%) players had at least one abnormality in their whole spine form (abnormal sagittal curve in thoracic or lumbar parts or scoliosis).

Thoracic spine kyphosis Cobb's angle mean (SD) of players with normal, hyper kyphosis, and straight back thoracic spine were: 26.15 (4.60), 37.35 (7.70) and 18.54 (3.39) respectively. Lumbar spine lordosis Cobb's angle mean (SD) of players with normal lumbar curve was 10 (1.14) and for players with hyper lordosis it was 21.02 (5.05).

\subsection{Nature of Lower Extremity Injuries}

Total number of players who suffered from lower extremity injuries were $n=111$ (45.49\% of total players), of which 78 players (31.97\% of total players) experienced at least one minor lower extremity injury (with $<28$ absence days) and 33 players (13.52\% of total players) suffered at least one major lower extremity injury (with $\geq 28$ absence days).

There were 155 injuries recorded in total with at least one day absence day from training or match, of which most common injuries were hamstring muscle injuries (n $=41,26 \%)$, ankle ligament injuries $(\mathrm{n}=34,22 \%)$ and adductor injuries ( $n=25,16 \%)$ respectively. The nature of these injuries is presented in Figure 1. 
Table 1. Records of Baseline Examinations

\begin{tabular}{|ll}
\hline Examination & Reporting Results \\
\hline Spine form & \\
\hline \multicolumn{1}{c}{ Thoracic spine } & Normal curve, hyper kyphotic, straight back \\
\hline Lumbar spine & Normal curve, hyper lordosis \\
\hline \multicolumn{1}{c}{ Coronal plane } & Normal, scoliosis \\
\hline Pelvic level & Even (0) or state the difference of right and left by cm \\
\hline Sacroiliac joint & Normal, abnormal \\
\hline Cervical rotation & \\
\hline \multicolumn{1}{c}{ Right } & Range of motion in degree \\
\hline \multicolumn{1}{c}{ Left } & State distance between fingertip and floor line, Positive numbers for hypo mobility, Negative numbers for crossing the floor line \\
\hline Spinal flexion & \\
\hline
\end{tabular}

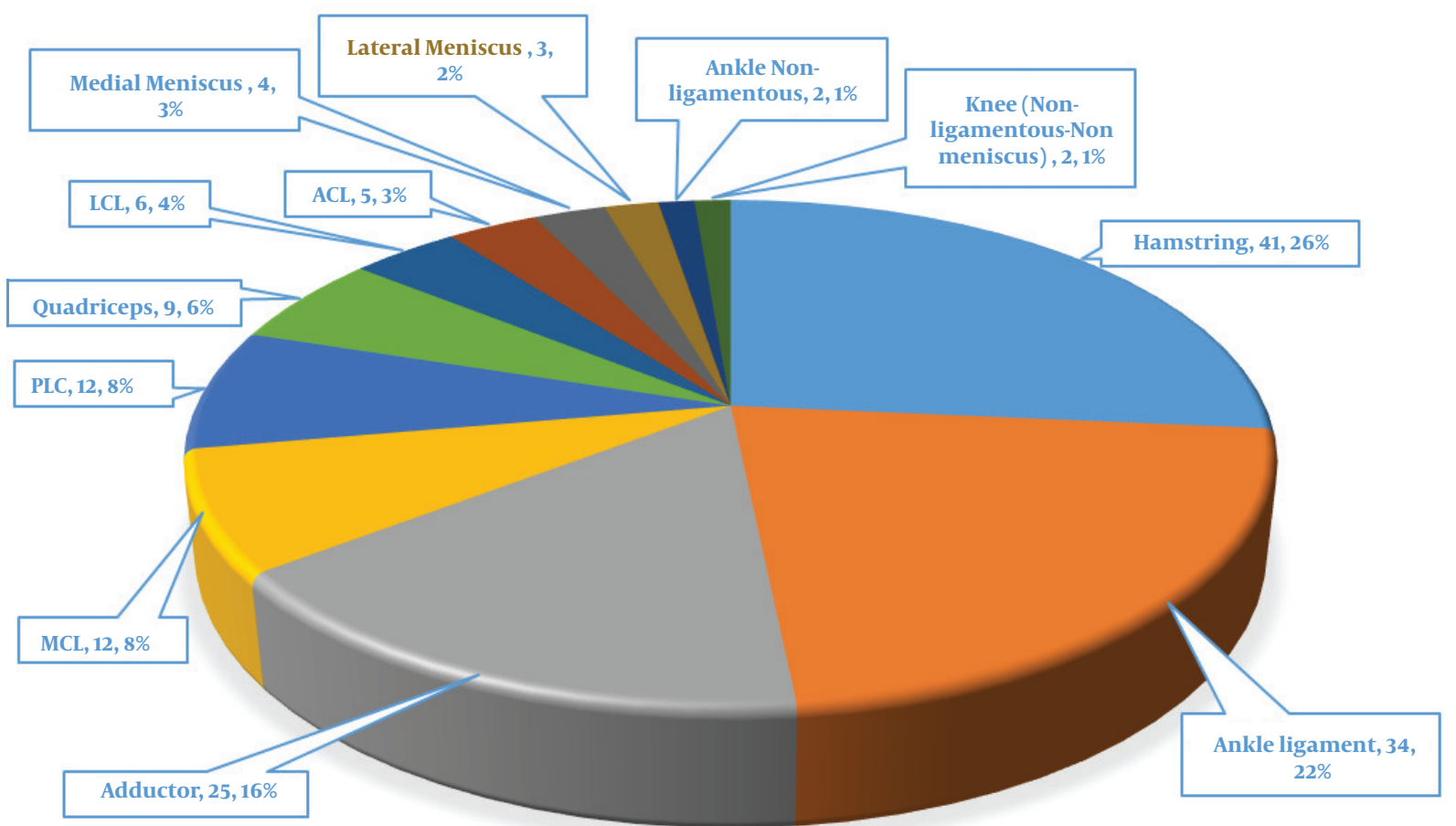

Figure 1. Nature of Lower Extremity Injuries

\subsection{Lower Extremity Injuries in Players with Abnormal Thoracic} Sagittal Curve

There was not a significant higher risk of lower extremity injuries among players with abnormal thoracic sagittal cure (hyper kyphosis or straight back). Relative risks (CI 95\%) of minor and major lower extremity injuries of players with abnormal thoracic sagittal curve are shown in Fig- ure 2.

3.4. Lower Extremity Injuries in Players With Hyper Lordosis Lumbar Sagittal Curve

There was a significant higher risk of major and minor lateral meniscus injuries among players with hyper lordosis of lumbar sagittal curve, RR (CI 95\%): 13.056 (1.301 


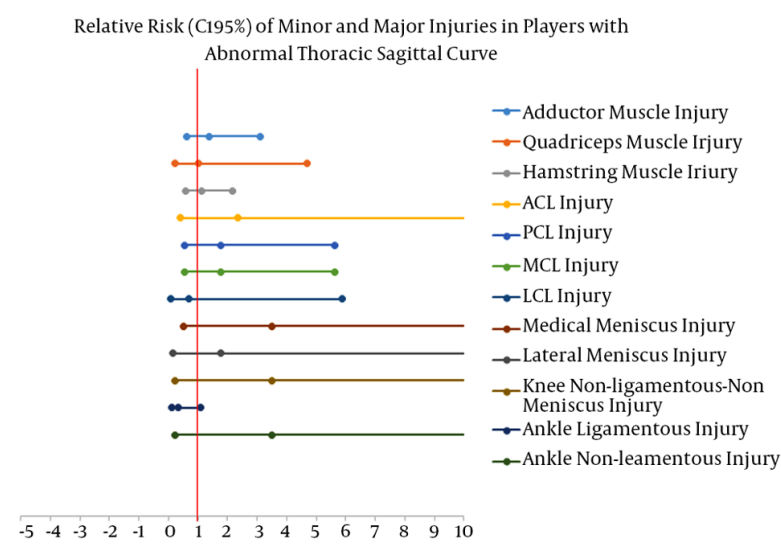

Relative Risk (C195\%) of Minor and Major Injuries in Players with

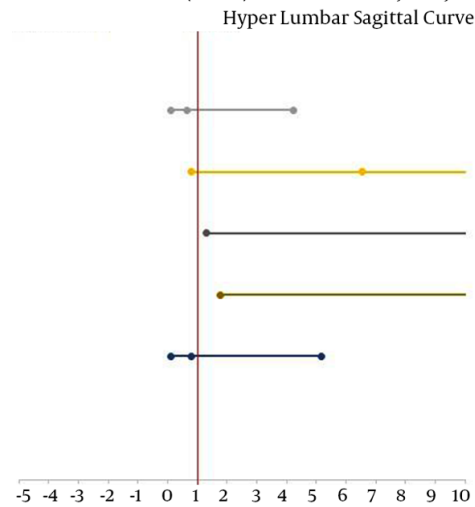

$\rightarrow$ Hamstring Muscle Iriury

$\rightarrow$ ACL Injury

$\rightarrow$ Lateral Meniscus Injury

$\rightarrow$-Knee Non-ligamentous-Non

Meniscus Injury

- Ankle Ligamentous Injury

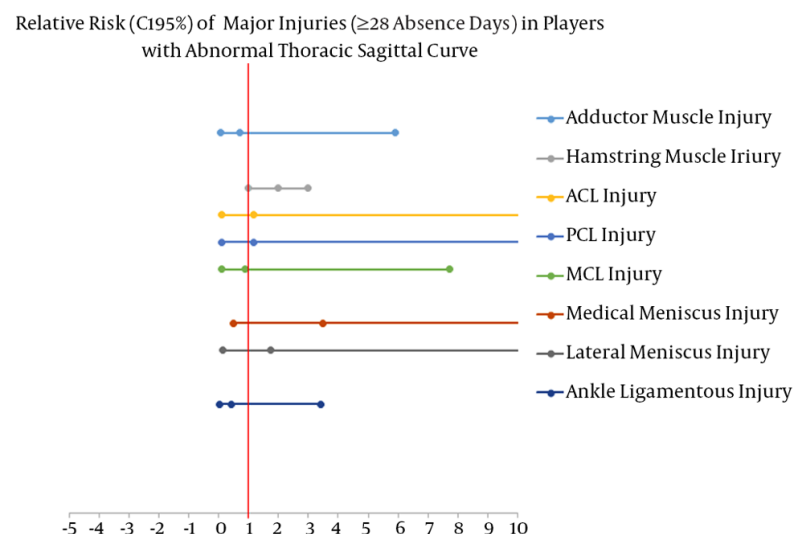

Relative Risk (C195\%) of Major Injuries ( $\geq 28$ Absence Days) in Players with Hyper Lumbar Sagittal Curve

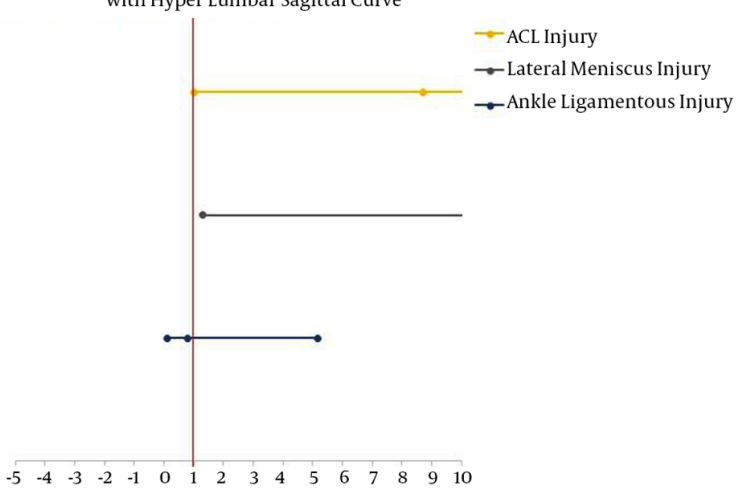

Figure 2. Relative Risks (CI95\%) of Lower Extremity Injuries of Player with Abnormal Thoracic Sagittal Curve (Hyper Kyphosis and Straight Back) and Hyper Lordotic Lumbar Sagittal Curve

Box 1. Classification of Injuries

\begin{tabular}{l}
\hline Classification of Injuries \\
\hline Adductor muscle injuries \\
\hline Quadriceps muscle injuries \\
\hline Hamstring muscle injuries \\
Knee injuries (ACL, PCL, MCL, LCL, medial meniscus, lateral meniscus, \\
non-ligamentous-non meniscus) \\
\hline Ankle injuries (ligamentous, non-ligamentous) \\
\hline
\end{tabular}

- 131.041). players with hyper lordosis also showed higher risk of major ( $>28$ days absence days) injury of lateral meniscus, RR (CI 95\%): 13.056 (1.301-131.041).

Risk of major and minor knee non-ligamententous non-meniscus injuries was higher among players with hyper lordosis of lumbar sagittal curve, RR (CI 95\%): 26.111 (1.771-384.921).
Major ( $>28$ days absence days) ACL injuries was more prevalent with RR (CI 95\%) of 8.704 (1.001 - 75.701) among players with hyper lordosis of lumbar sagittal curve.

There was no other significant higher risk of lower extremity injuries among players with hyper lordosis of sagittal curve. Valid relative risks and 95\% CI s are shown in Figure 2.

3.5. Lower Extremity Injuries in Players with Scoliosis and Sacroiliac Joint Abnormality

Players with scoliosis $(\mathrm{n}=3$ ) or sacroiliac joint abnormality $(\mathrm{n}=2)$ experienced no lower extremity injury, therefore relative risks were not calculated.

\subsection{Lower Extremity Injuries and Continuous Independent Vari-} ables

3.6.1. Cobb's Angles

Logistic regression was performed to ascertain the effects of thoracic and lumbar Cobb's angle on the likelihood 


\begin{tabular}{|c|c|}
\hline Variables & Value \\
\hline \multicolumn{2}{|l|}{ Spine Form } \\
\hline \multicolumn{2}{|l|}{ Thoracic Spine } \\
\hline Normal curve & $77.9(190)$ \\
\hline Hyper kyphotic & $18.9(46)$ \\
\hline Straight back & $3.3(8)$ \\
\hline \multicolumn{2}{|l|}{ Lumbar Spine } \\
\hline Normal curve & $96.3(235)$ \\
\hline Hyper lordosis & $3.7(9)$ \\
\hline \multicolumn{2}{|l|}{ Coronal Plane } \\
\hline Normal & $98.8(242)$ \\
\hline Scoliosis & $1.2(2)$ \\
\hline \multicolumn{2}{|l|}{ Sacroiliac Joint } \\
\hline Normal & $99.2(241)$ \\
\hline Abnormal & $0.8(3)$ \\
\hline
\end{tabular}

of minor and major lower extremity injuries.

For major and minor total injury, the logistic regression model was statistically significant $\chi^{2}(9)=20.45$, P $=0.015$.The model explained 10\% (Nagelkerke $\mathrm{R}^{2}$ ) of the variance in total lower extremity injury (major and minor) and correctly classified $58.2 \%$ of cases. Increasing thoracic kyphosis Cobb's angle was associated with a reduction in the likelihood of exhibiting minor and major lower extremity injury, OR (CI 95\%): 0.947 (0.901 - 0.994) (Table 3).

\subsubsection{Thoracic Kyphosis Cobb's Angle and Quadriceps Injuries}

For major and minor quadriceps injury, the logistic regression model was statistically significant $\chi^{2}(9)=19.33$, $\mathrm{P}=0.022$.The model explained 28\% (Nagelkerke $\mathrm{R}^{2}$ ) of the variance in quadriceps injury (major and minor) and correctly classified $96.7 .0 \%$ of cases. Increasing thoracic kyphosis Cobb's angle was associated with a reduction in the likelihood of exhibiting minor and major quadriceps injury, OR (CI 95\%): 0.806 (0.671 - 0.968) (Table 3)

There was no other statically significant model which shows association between thoracic and lumbar lordosis Cobb's angle and lower extremity injuries (Tables 3 and 4 ).

\subsubsection{Cervical Spine Range of Motion, Spinal Flexion and Pelvic Level}

No statistically significant logistic model showed association between lower extremity injuries and cervical spine range of motion, spinal flexion and pelvic level ( $P$ value $>0.05$ ) (Tables 3 and 4 ).

\section{Discussion}

\subsection{Hyper Lordosis Lumbar Sagittal Curve}

This study identified hyper lordosis lumbar sagittal curve as a risk factor for major ACL and also major and minor lateral meniscus and knee non-ligamentous and nonmeniscal injuries.

Watson suggested before that knee injuries are associated with lumbar lordosis (13). Watson reported that $67 \%$ of players with lumbar hyper lordosis suffered the injuries, while in group with normal lumbar lordosis only $36 \%$ suffered the injury. He reported that $51.9 \%$ of college football players presented with lumbar lordosis. Lumbar hyper lordosis had lower prevalence (9\%) among our subjects; this lower prevalence can be attributed to the elite level of our subjects.

Our prospective study showed that ACL and lateral meniscus of knee are more susceptible to injuries among players with hyper lordosis. This suggests that pre participation examinations of player's lumbar sagittal curve could be of value to reduce knee injuries.

Another cross sectional study by Hennessey and Watson, among 34 cases which investigated posture, including lumbar lordosis in athletes with and without history of hamstring injury. The results of the study indicated that while differences in hamstring flexibility were not evident between injured and noninsured groups, poorer low back posture was found in the injured group (20). But in our longitudinal study lumbar hyper lordosis was not a predictor for hamstring injury.

Any increased kyphosis in lumbar spine results in compensation, first as rotation in the pelvis and then knee flexion in the lower limbs (21). This compensation maintains the line of gravity inside the base of support but is not ergonomic. Compensatory changes in the knees, pelvis, and spine, cause higher energy expenditure and also increased muscular forces and fatigue. Mair et al. (22) showed that fatigued muscles are less capable of absorbing energy before reaching the point of stretch that leads to injuries.

Another explanation for association between lumbar hyper lordosis and lower exterimity injuries is through core stability. Lumbopelvic or core stability is the ability of the lumbopelvic hip complex to prevent buckling and to return to equilibrium after perturbation. Static elements (bone and soft tissue) contribute to core stability. There is a clear relationship between core stability and lower extremity movement. Current evidence suggests that decreased core stability may predispose to injury (12).

\subsection{Thoracic Sagittal Curve Cobb's Angle}

Another interesting finding was that increasing thoracic kyphosis Cobb's angle is a protective factor for 
Table 4. Analysis of Risk Factor Variables for Major (> 4 Weeks) Lower Extremity Injuries from Binary Logistic Regression ${ }^{\mathrm{a}}$

\begin{tabular}{|c|c|c|c|c|c|c|c|c|c|c|c|c|}
\hline \multirow[t]{3}{*}{ Variable } & \multicolumn{12}{|c|}{ Type of Injuries } \\
\hline & \multicolumn{2}{|c|}{ Adductor } & \multicolumn{2}{|c|}{ Quadriceps } & \multicolumn{2}{|c|}{ Hamstring } & \multicolumn{2}{|c|}{ Knee } & \multicolumn{2}{|c|}{ Ankle } & \multicolumn{2}{|c|}{ Total Lower Extremity } \\
\hline & OR & PValue & OR & PValue & OR & PValue & OR & PValue & OR & PValue & OR & PValue \\
\hline Spine form abnormality & 0.71 & 0.60 & $>10$ & 0.99 & $>10$ & 0.99 & 0.78 & 0.68 & $>10$ & 0.99 & 0.44 & 0.47 \\
\hline Thoracic kyphosis & $>10$ & 0.99 & 0.47 & 0.99 & $<0.1$ & 0.99 & 0.78 & 0.73 & $<0.1$ & 0.99 & 4.90 & 0.22 \\
\hline Lumbar lordosis & $<0.1$ & 0.99 & $<0.1$ & 0.99 & 2.11 & 0.99 & 2.76 & 0.50 & 0.15 & 0.99 & 0.97 & 0.99 \\
\hline Scoliosis & $>10$ & 0.99 & 0.73 & 0.99 & 0.95 & 0.99 & $>10$ & 0.99 & 0.82 & 0.99 & $<0.1$ & 0.99 \\
\hline \multicolumn{13}{|l|}{ Curves } \\
\hline Thoracic Cobb's & 0.86 & 0.14 & 0.87 & 0.44 & 0.99 & 0.96 & 0.93 & 0.21 & 0.79 & $<0.1$ & 0.90 & 0.00 \\
\hline Lumbar Cobb's & 1.02 & 0.94 & 0.42 & 0.25 & 1.14 & 0.67 & 1.21 & 0.30 & 0.78 & 0.44 & 1.06 & 0.66 \\
\hline Pelvic Level & $<0.1$ & 0.99 & $<0.1$ & 0.99 & $<0.1$ & 0.99 & 1.18 & 0.85 & 4.78 & 0.04 & 1.58 & 0.42 \\
\hline \multicolumn{13}{|l|}{ Neck spine range of motion } \\
\hline Right & 1.46 & 0.99 & 1.41 & 0.99 & 1.47 & 0.99 & 1.50 & 0.99 & 1.47 & 0.99 & 1.53 & 0.99 \\
\hline left & 1.72 & 0.99 & 1.56 & 0.99 & 1.42 & 0.99 & 1.52 & 0.99 & 1.43 & 0.99 & 1.52 & 0.99 \\
\hline Spinal flexion & 0.90 & 0.47 & 0.89 & 0.65 & 0.84 & 0.18 & 1.02 & 0.99 & 1.01 & 0.84 & 0.98 & 0.69 \\
\hline Total model & & 0.52 & & 0.90 & & 0.81 & & 0.85 & & 0.22 & & 0.34 \\
\hline
\end{tabular}

${ }^{\mathrm{a}}$ No player had major non ligament non meniscus knee injury and No player had major non ligament ankle injury.

quadriceps injury. To our knowledge our study is the first study that investigated and showed association between thoracic spine curve and lower extremity injuries.

The reason why players with lower thoracic kyphosis Cobb's angle may be at risk for quadriceps injury is not clear. But biomechanical changes such as extensibility of hamstring and the balance between hamstring and quadriceps may partially explain the increased risk. This is consistent with previous studies which showed the effect of hamstring stretching on sagittal spinal curvatures especially decreasing thoracic kyphotic curve (23). It may imply that higher thoracic kyphosis can have a protective effect on quadriceps injury through tightness of hamstring. Further studies can investigate that if thoracic kyphosis is protective for quadriceps injuries in both players with and without hamstring tightness.

These findings can be used in developing new injury preventing strategies.

\subsection{Methodological Considerations}

Limitations of this study should be acknowledged. First the definition and classification of each part injury, injuries constitute a heterogeneous group including strains, sprains, contusions and overuse injuries. We used a practical approach to register injuries, and the fact that many club doctors were involved in injury diagnosis and the nature of clinical diagnosis without obligatory radiological verification may decrease the reliability of injury records. Nevertheless, our data are based on clinical data reported by experienced club doctors who were trained and certified by Iranian Football Federation medical committee and were often confirmed with radiological examination. Injuries with an acute or gradual onset may have different origins, and this was not reflected in our data set. A further subgrouping of injuries according to grade, location, and measurements of pathology may be useful when studying risk factors for lower extremity injuries. Most of the risk factors studied in our study were not modifiable or hard to modify, for example, spine form and scoliosis. However, awareness of such factors may still be of value to classify subgroups of players at increased risk of injury.

More studies on possible mechanism of effect of lumbar lordosis on knee injuries and thoracic kyphosis' preventive effect on quadriceps injuries would be of great value to improve preventive measures and to decrease the burden of lower extremity injuries in football.

We should also mention that lumbar lordosis, thoracic kyphosis and cervical alignment are closely related to pelvic incidence as a pelvic parameter, further studies on the relationship of spine and lower extremity injuries should consider this part of the spinal chain.

Our study showed the link of spine form with lower extremity injuries in both a protective and hazardous way. Further studies should concentrate on possible mechanisms of this association.

\section{Acknowledgments}

We should thank all the club doctors of Iranian Premier Football League for their cooperation through the one year of this study. 


\section{Footnote}

Financial Disclosure: There is no conflict of interest for any author regarding this manuscript.

\section{References}

1. Kunz M. 265 million playing football. FIFA Magazin. 2007:10-5.

2. Arliani GG, Belangero PS, Runco JL, Cohen M. The Brazilian Football Association (CBF) model for epidemiological studies on professional soccer player injuries. Clinics (Sao Paulo). 2011;66(10):1707-12. [PubMed: 22012041].

3. Ekstrand J, Hagglund M, Walden M. Injury incidence and injury patterns in professional football: the UEFA injury study. Br J Sports Med. 2011;45(7):553-8. doi: 10.1136/bjsm.2009.060582. [PubMed: 19553225].

4. Caine DJ, Maffulli N. Epidemiology of pediatric sports injuries. Karger Medical and Scientific Publishers; 2005.

5. Arnason A, Sigurdsson SB, Gudmundsson A, Holme I, Engebretsen L, Bahr R. Risk factors for injuries in football. Am J Sports Med. 2004;32(1 Suppl):5S-16S. doi: 10.1177/0363546503258912. [PubMed:14754854].

6. Hagglund M, Walden M, Ekstrand J. Previous injury as a risk factor for injury in elite football: a prospective study over two consecutive seasons. $\mathrm{Br} J$ Sports Med. 2006;40(9):767-72. doi: 10.1136/bjsm.2006.026609. [PubMed:16855067].

7. Lundblad M, Walden M, Hagglund M, Ekstrand J, Thomee C, Karlsson J. No Association Between Return to Play After Injury and Increased Rate of Anterior Cruciate Ligament Injury in Men's Professional Soccer. Orthop J Sports Med. 2016;4(10):2325967116669708. doi: 10.1177/2325967116669708. [PubMed: 27830159].

8. Bradley PS, Portas MD. The relationship between preseason range of motion and muscle strain injury in elite soccer players. J Strength Cond Res. 2007;21(4):1155-9. doi:10.1519/R-20416.1. [PubMed: 18076233].

9. Witvrouw E, Danneels L, Asselman P, D’Have T, Cambier D. Muscle flexibility as a risk factor for developing muscle injuries in male professional soccer players. A prospective study. Am J Sports Med. 2003;31(1):41-6. doi: 10.1177/03635465030310011801. [PubMed: 12531755].

10. Engebretsen AH, Myklebust G, Holme I, Engebretsen L, Bahr R. In trinsic risk factors for groin injuries among male soccer players: prospective cohort study. Am J Sports Med. 2010;38(10):2051-7. doi 10.1177/0363546510375544. [PubMed: 20699426].

11. Steffen K, Nilstad A, Kristianslund EK, Myklebust G, Bahr R, Krosshaug T. Association between Lower Extremity Muscle Strength and Noncontact ACL Injuries. Med Sci Sports Exerc. 2016;48(11):2082-9. doi: 10.1249/MSS.0000000000001014. [PubMed: 27327027].
12. Willson JD, Dougherty CP, Ireland ML, Davis IM. Core stability and its relationship to lower extremity function and injury.J Am Acad Orthop Surg. 2005;13(5):316-25. [PubMed: 16148357].

13. Watson AW. Sports injuries in footballers related to defects of posture and body mechanics. J Sports Med Phys Fitness. 1995;35(4):289-94. [PubMed: 8776077].

14. Kordi R, Hemmati F, Heidarian H, Ziaee V. Comparison of the incidence, nature and cause of injuries sustained on dirt field and artificial turf field by amateur football players. Sports Med Arthrosc Rehabil Ther Technol. 2011;3:3. doi: 10.1186/1758-2555-3-3. [PubMed: 21306640].

15. Dvorak J, Grimm K, Schmied C, Junge A. Development and implementation of a standardized precompetition medical assessment of international elite football players-2006 FIFA World Cup Germany. Clin J Sport Med. 2009;19(4):316-21. doi: 10.1097/JSM.0b013e3181b21b6e. [PubMed: 19638827].

16. Dvorak J, Junge A, Grimm K. F-MARC football medicine manual. : FIFA; 2009. 2nd ed. Zurich: FIFA Medical Assessment and Research Centre (F-MARC); 2009. p. 254.

17. Cobb J. Outline for the study of scoliosis: Instructional course lecture 5. Sci Res. 1948;5:261-75.

18. Mannion AF, Knecht K, Balaban G, Dvorak J, Grob D. A new skin-surface device for measuring the curvature and global and segmental ranges of motion of the spine: reliability of measurements and comparison with data reviewed from the literature. Eur Spine J. 2004;13(2):122-36. doi: 10.1007/s00586-003-0618-8. [PubMed: 14661104].

19. Fuller CW, Ekstrand J, Junge A, Andersen TE, Bahr R, Dvorak J, et al. Consensus statement on injury definitions and data collection procedures in studies of football (soccer) injuries. Br J Sports Med. 2006;40(3):193-201. doi: 10.1136/bjsm.2005.025270. [PubMed: 16505073].

20. Hennessey L, Watson AW. Flexibility and posture assessment in relation to hamstring injury. Br J Sports Med. 1993;27(4):243-6. [PubMed: 8130961].

21. Le Huec JC, Saddiki R, Franke J, Rigal J, Aunoble S. Equilibrium of the human body and the gravity line: the basics. Eur Spine J. 2011;20 Suppl 5:558-63. doi: 10.1007/s00586-011-1939-7. [PubMed: 21809013].

22. Mair SD, Seaber AV, Glisson RR, Garrett WJ. The role of fatigue in susceptibility to acute muscle strain injury. Am J Sports Med. 1996;24(2):137-43. doi: 10.1177/036354659602400203. [PubMed: 8775109].

23. Lopez-Minarro PA, Muyor JM, Belmonte F, Alacid F. Acute effects of hamstring stretching on sagittal spinal curvatures and pelvic tilt. $J$ Hum Kinet. 2012;31:69-78. doi: 10.2478/v10078-012-0007-7. [PubMed: 23486214]. 


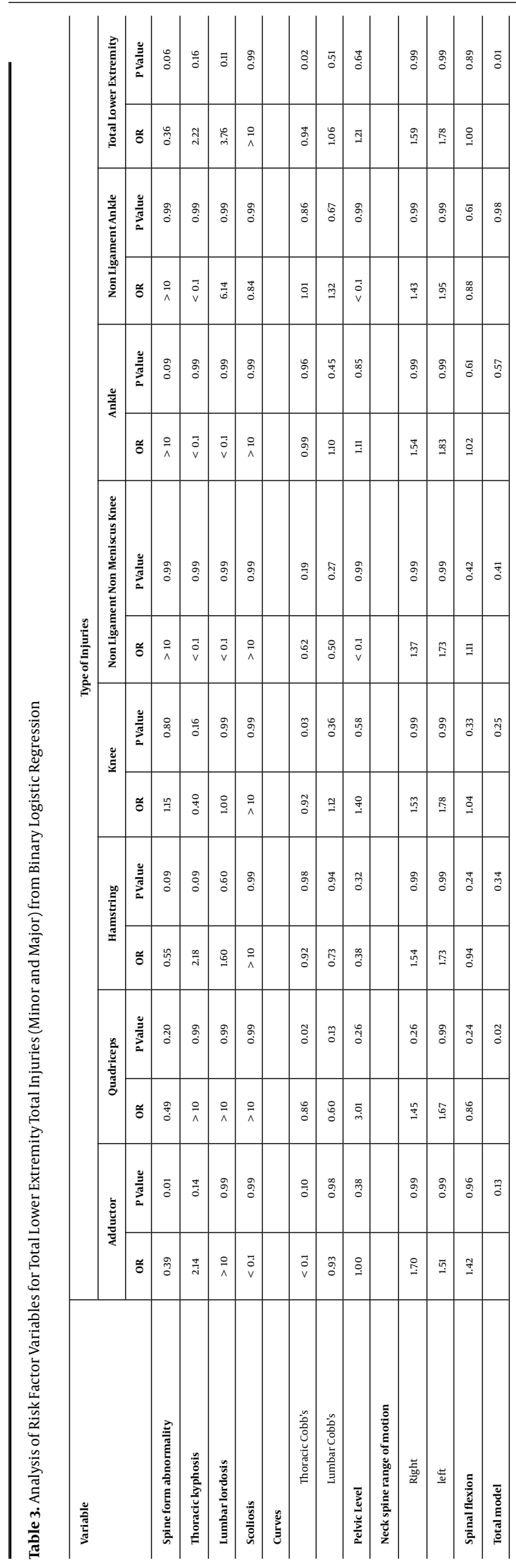

